\title{
Genetic predisposition to multiple myeloma
}

\author{
Yan Cheng ${ }^{1}$, Fumou Sun ${ }^{1}$, Xing Cui ${ }^{2}$, Siegfried Janz ${ }^{1 *}$ \\ ${ }^{I}$ Division of Hematology and Oncology, Medical College of Wisconsin, Milwaukee 53226, WI, USA; \\ ${ }^{2}$ Department of Hematology, Affiliated Hospital of Shandong University of Traditional Chinese Medicine, Jinan, Shandong 250014, China.
}

\begin{abstract}
Genetic myeloma risk research relied on genome-wide association studies to identify 24 common but lowimpact germline predisposition alleles that account for an estimated one eighth of the heritable myeloma risk in Caucasians. Next-generation sequencing, particularly whole-exome sequencing, uncovered a handful of rare but high-impact myeloma risk loci that convey intriguing clues about etiology. The recent discovery of NCOAl as a myeloma susceptibility gene in Han Chinese has set the stage for the more complete elucidation of the genetic myeloma risk across ethnic barriers. Validating individual myeloma risk loci at the functional level and integrating predisposition genes in genetic networks and biological pathways are important research tasks going forward. Candidate pathways that are currently emerging include plasma cell development, autophagy, telomere maintenance, and cell cycle regulation. An outstanding knowledge gap in the area of gene-environment interaction concerns the possibility that tumor-promoting effects of myeloma susceptibility alleles depend on specific environmental or occupational exposures. An implicit promise of myeloma risk research is the detection of new molecular targets for myeloma treatments and preventions. A related outcome is new biomarkers for patient stratification, prognostication, and development of individualized treatment plans.
\end{abstract}

Keywords: plasma cell malignancy, germline risk, racial and ethnic factors, GWAS

Abbreviations: GWAS, genome-wide association study; MGUS, monoclonal gammopathy of undetermined significance; MM, multiple myeloma; NGS, next-generation sequencing; OR, odds ratio; RAF, relative allele frequency; RR, relative risk; TWAS, transcriptome-wide association study; WES, whole exome sequencing

\section{DISCOVERY OF GENETIC MYELOMA RISK}

The discovery of genetic predisposition to multiple myeloma (MM) goes back almost exactly a century ago to the 1920s, when for the first time families were described in which several members were affected by MM (familial myeloma) or its precursor condition, monoclonal gammopathy of undetermined significance (MGUS). Recent epidemiological case control studies have brought these early observational findings to date, including a large Swedish survey published in 2010, which analyzed nearly 14 thousand myeloma patients and more than fifty thousand healthy controls. The analysis revealed that first-degree relatives of myeloma patients had a 2.1-fold higher relative risk (RR) of developing both MM and MGUS ${ }^{[1]}$. RR of acute lymphoblastic leukemia was also increased by a factor of 2.1, suggesting that myeloma risk overlaps with that of less mature B-lineage neoplasms $^{[1]}$. Hypothesis-driven genetic association

*Correspondence to: Siegfried Janz, Division of Hematology and Oncology, Medical College of Wisconsin, Milwaukee 53226, WI, USA. TEL: +1414-955-5784, E-mail: sjanz@mcw.edu.

The authors declared no conflict of interests. 
studies that utilized a candidate gene or pathway approach to identify genetic variants that elevate myeloma risk complemented these investigations. This included polymorphisms in genetic networks one might intuitively implicate in the natural history of myeloma; e.g., cytokine-dependent immune responses, DNA repair, and apoptosis. However, less obvious connections, such as folate metabolism and insulin-like growth factor signaling, were also considered. A number of positive associations with myeloma risk were suspected, but none of these were independently replicated or free of (potentially fatal) flaws such as insufficient sample size or cryptic relatedness of probands ${ }^{[2]}$. The recent technological advance of GWAS, a method of unbiased genetic association testing, permitted the field to overcome these shortcomings and identify, for the first time, myeloma risk loci in a definitive fashion. Fig. 1 depicts a brief timeline of myeloma risk studies, including ongoing research aimed at annotating genetic risk with biological functionality. The prospective epidemiologic PROMISE study, which attempts to predict myeloma progression in high-risk individuals, is also included. This review will summarize available findings on myeloma risk and identify knowledge gaps that should be addressed in future work. Included in the latter are racial and ethnic differences in myeloma risk between Han Chinese and people of Caucasian and African ancestry.

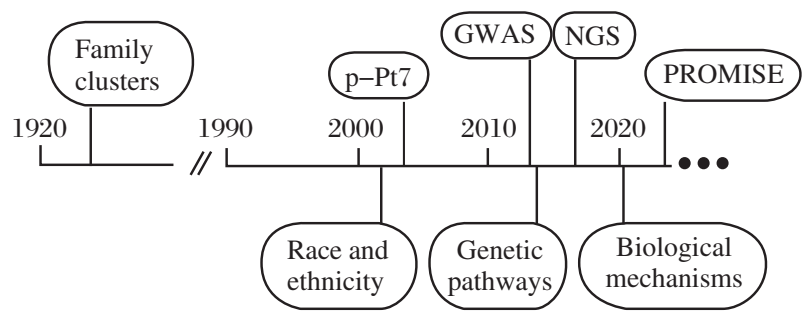

Fig. 1 Milestones of inherited myeloma risk research. Depicted is a timeline of discovery that began with the detection of familial myeloma a century ago and took a leap forward in the new century with the introduction of GWAS (genome-wide association study) and NGS (next generation sequencing). GWAS and NGS have uncovered common, low-impact and rare, high-impact risk alleles, respectively. PROMISE stands for Predicting Progression of Developing Myeloma in a High-Risk Screened Population.

\section{MYELOMA RISK ALLELES IDENTI- FIED BY GWAS}

GWAS, also known as whole genome association study or WGAS, is an observational method for assessing a genome-wide set of genetic variants (typically single-nucleotide polymorphisms or SNPs) in different individuals (e.g., patients $v s$. healthy controls) in order to determine whether any of these variants are associated with a particular trait such as susceptibility to MM. One strength of GWAS is the ability to achieve a stringent threshold of genomewide significance $\left(5 \times 10^{-8}\right.$ is usually required) and its flexibility in terms of considering independent study results for candidate locus confirmation. Beginning in 2012, GWAS identified myeloma susceptibility loci on chromosomes $3 \mathrm{p} 22,7 \mathrm{p} 15.3$, $8 \mathrm{q} 24$ and $2 \mathrm{p} 23.3^{[3,4]}$, with relative allele frequencies (RAFs) and per-allele odds ratios (ORs) invariably indicating common and low-risk variants (Table 1). By 2016 additional GWAS and case-control studies had uncovered association signals for 17 risk variants ${ }^{[5-7]}$, a number that could be increased to 24 in the most recent reports ${ }^{[8,9]}$. Some risk variants; e.g., located at 16p13, are also associated with survival of patients with myeloma ${ }^{[10]}$. Despite the leap forward made possible by GWAS, much of the heritable risk of myeloma remains unexplained as of today--a widely known and extensively discussed shortcoming of the method that perpetrates the entire cancer field. The 24 loci mentioned above explain but an estimated one eighth of the heritability for myeloma in Caucasians, with estimates that a sample size in excess of $5 \times 10^{4}$ is required to explain $80 \%$ of the heritability ${ }^{[8]}$. Some genetic risk variants exhibit myeloma subtype-specific preference; e.g., a variant at $C C N D 1$ (cyclin $\mathrm{D} 1$, required for cell cycle $\mathrm{Gl} / \mathrm{S}$ transition) is associated with $\mathrm{t}(11 ; 14)$-harboring myeloma $^{[5]}$, whereas a variant at $C B X 7$ (chromobox 7 , a transcriptional repressor) is linked to myeloma that does not carry the cyclin Dl-deregulating chromosomal translocation ${ }^{[6]}$. Subtype associations of this sort are of interest for our working model on myelomagenesis because they point to independent pathways of tumor development distinguished by different cytogenetic and molecular features ${ }^{[11]}$.

\section{MYELOMA RISK OVERLAPS WITH RISK TO MGUS AND AL AMYLOIDOSIS}

In parallel to elucidating heritable myeloma risk, GWAS has been successfully employed to delineate the genetic risk of AL (immunoglobulin lightchain) amyloidosis, a closely related neoplasm, and the premalignant condition, MGUS ${ }^{[12]}$. Unsurprisingly, the underlying genetic risk loci exhibit major overlap ${ }^{[13]}$. The most recent meta-analysis of MM $(n=4,403)$, AL amyloidosis $(n=1,230)$ and MGUS $(n=992)$, revealed 17 independent loci ${ }^{[14]}$, nine of which are included in Table 1 (indicated by asterisks in the first column) because they were 
Table 1 Myeloma risk variants discovered by GWAS and confirmed, in part, by TWAS

\begin{tabular}{|c|c|c|c|c|c|c|}
\hline $\begin{array}{l}\text { Genetic } \\
\text { locus }^{1}\end{array}$ & $\mathrm{RAF}^{2}$ & $\mathrm{OR}^{3}$ & GWAS candidate gene $^{4}$ & Ref. $^{5}$ & Meta-anal. $^{6}$ & TWAS candidate gene $^{7}$ \\
\hline $2 \mathrm{p} 23.3^{*}$ & 0.81 & 1.24 & DNMT3A, DTNB & {$[3,7]$} & $\checkmark$ & $\begin{array}{l}\text { CENPO, DNAJC27, DNMT3A, } \\
\text { DTNB, EPT1, KIF3C, PTGES3P2 }\end{array}$ \\
\hline $2 \mathrm{q} 31.1$ & 0.77 & 1.12 & $S P 3$ & [8] & - & - \\
\hline $3 \mathrm{p} 22.1^{*}$ & 0.16 & 1.26 & ULK4 & {$[3,7]$} & $\sqrt{ }$ & ULK4 \\
\hline $3 \mathrm{q} 26.2^{*}$ & 0.75 & 1.20 & $\begin{array}{l}\text { ACTRT3, GPR160, MYNN, PDCD10, LRRC31, } \\
\text { LRRC34, PDCD10, PHC3, SAMD7, SEC62, } \\
\text { SEC62-AS1, SERPINI1, SKIL, TERC }\end{array}$ & {$[6-8]$} & $\sqrt{ }$ & $\begin{array}{l}\text { ACTRT3, MYNN, LRRC34, } \\
\text { LRRIQ4 }\end{array}$ \\
\hline $5 q 15$ & 0.75 & 1.17 & ELL2, VPS13C & {$[7,58]$} & $\sqrt{ }$ & - \\
\hline $5 q 23.2$ & 0.43 & 1.11 & CEP120, SNX2, SNX24 & [8] & - & - \\
\hline $6 \mathrm{p} 21.3^{*}$ & 0.29 & 1.20 & CCHCRl, CDSN, POU5F1, PSORS1Cl, TCF19 & {$[6-8]$} & $\sqrt{ }$ & - \\
\hline $6 \mathrm{p} 22.3$ & 0.02 & 1.37 & JARID2 & {$[7]$} & $\sqrt{ }$ & - \\
\hline $6 q 21$ & 0.21 & 1.18 & ATG5, PRDM1, PREP & {$[7,8]$} & $\sqrt{ }$ & ATG5 \\
\hline $7 \mathrm{p} 15.3^{*}$ & 0.65 & 1.38 & CDCA7L, DNAHI1 & {$[3,37]$} & $\sqrt{ }$ & CDCA7L \\
\hline $7 q 22.3$ & 0.74 & 1.12 & $C C D C 71 L$ & [8] & - & - \\
\hline $7 \mathrm{q} 31.33$ & 0.72 & 1.12 & $A S B 15, I Q U B, P O T 1$, WASL & [8] & - & - \\
\hline $7 \mathrm{q} 36.1^{*}$ & 0.12 & 1.19 & ABCF2, ASIC3, ATG98, CHPF2, SMARCD3 & [7] & $\sqrt{ }$ & CHPF2 \\
\hline $8 \mathrm{q} 24.21$ & 0.32 & 1.13 & CASC11, CCAT1, MYC & {$[7,8]$} & $\sqrt{ }$ & - \\
\hline $9 \mathrm{p} 21.3^{*}$ & 0.63 & 1.15 & $C D K N 2 A, C D K N 2 B-A S 1, M T A P$ & [7] & $\sqrt{ }$ & - \\
\hline 10p12.1 & 0.73 & 1.12 & LYZL1, MASTL, WAC, YME1L1 & {$[7,8]$} & $\sqrt{ }$ & - \\
\hline $11 \mathrm{q} 13.3$ & 0.51 & 1.82 & CCND1 & [5] & - & - \\
\hline $16 \mathrm{p} 11.2$ & 0.27 & 1.15 & $\begin{array}{l}D C T P P 1, D O C 2 A, F B R S, F B X L 19, \text { GDPD3, ITGAL, } \\
\text { MYLPF, PPP4C, PRR14, RNF40, SEPHS2, SEPT1, } \\
\text { SRCAP, TBCID1OB, ZNF48, ZNF771 }\end{array}$ & {$[8]$} & - & $\begin{array}{l}\text { C16orf93, PRR14, PRSS53, QPRT, } \\
\text { RNF40, RP11-2C24.5 }\end{array}$ \\
\hline 16q23.1 & 0.58 & 1.13 & $\begin{array}{l}\text { CFDP1, GABARAPL2, GLG1, HSPE1P, NPIPL2, } \\
\text { PSMD7, RFWD3 }\end{array}$ & [7] & $\checkmark$ & $R F W D 3$ \\
\hline $17 \mathrm{p} 11.2^{*}$ & 0.10 & 1.30 & TNFRSF13B & [6] & $\sqrt{ }$ & PEMT, TBC1D27, USP32P1 \\
\hline 19p13.11 & 0.24 & 1.14 & $K L F 2$ & {$[8]$} & - & - \\
\hline $20 q 13.13$ & 0.08 & 1.26 & ARFGEF2, PREXI & {$[7,8]$} & $\sqrt{ }$ & - \\
\hline $22 \mathrm{q} 13$ & 0.37 & 1.21 & $\begin{array}{l}\text { APOL3, CRYBB1, FBXO7, HMGXB4, HMOX1, } \\
L A R G E, M B, R A S D 2, T O M 1\end{array}$ & {$[8,58]$} & $\sqrt{ }$ & - \\
\hline $22 \mathrm{q} 13.1^{*}$ & 0.44 & 1.23 & APOBEC $3 B-A S 1, C B X 7, R P L 3$ & {$[6,8]$} & $\sqrt{ }$ & 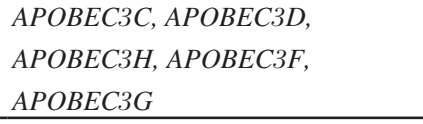 \\
\hline
\end{tabular}

${ }^{1}$ Chromosomal location of myeloma risk locus identified in genome-wide association study, or GWAS. Asterisk indicates locus is also associated with risk of MGUS and AL amyloidosis.

${ }^{2}$ Relative allele frequency reported by Went et al. $2018^{[8]}$. Mean value of RAFs: 0.445 . Standard deviation: 0.255. Range: $0.02-0.81$.

${ }^{3}$ Odds ratio reported by Went et al. $2018^{[8]}$. Indicates that carrier status of risk allele increases the odds of developing myeloma from $11 \%$ (5q23.2) to $82 \%$ (11q13.3). Mean value of ORs: 1.22. Standard deviation: 0.146 .

${ }^{4}$ Gene containing the risk SNP within or outside the gene body. In case of the latter, evidence indicates genetic interaction affects gene expression. Bold, confirmed by transcriptome-wide association study, or TWAS ${ }^{[59]}$.

${ }^{5}$ References.

${ }^{6}$ Confirmed in recent meta-analysis carried out by Pertesi and associates ${ }^{[9]}$.

${ }^{7}$ Myeloma risk locus identified by transcriptome-wide association study, or TWAS. Bold, confirmed by genome-wide association study, or GWAS.

previously identified in GWA studies on MM. Eight loci were newly identified (not included in the table) and pointed to candidate genes on chromosomes 2 (ASXL2, KIF3C), $4(R P 11-818 C 3.1), 5$ (ARHGAP26, GABRA1, GABRG2), 6 (HLA-DRA), 8 (TOX-CA8), 11 (B4GALNT4) and 13 (TPTE2). Some of these genes are intriguing from a tumor development point-of-view; e.g., ASXL2 (ASXL transcriptional regulator 2) at $2 \mathrm{p} 23.3$, because it is required for normal hematopoiesis and can function as a haplo-insufficient tumor suppressor, and ARHGAP26 (Rho GTPase activating protein 26) because it is a fusion partner of $M L L$ (mixed-lineage leukemia, officially designated KMT2A or lysine methyltransferase 2A), an important driver of leukemia. Genetic interaction--a newly developed approach to accomplish both annotating genetic risk patterns with biological functionality and integrating variant pair interaction with genetic network and pathway enrichment analysis-directed attention to $B$ cell receptor (BCR) signaling regulated by PREX1 (phosphatidylinositol-3,4,5-trisphosphate dependent Rac exchange factor 1) and SETBP1 (SET binding protein 1) as putative drivers of neo- 
plastic plasma cell transformation ${ }^{[15]}$. Of note, the overall estimated heritability of MGUS (25\%) that can be explained by the sum of all genetic risk alleles identified to date is approximately twice as high as that of MM (13\%) and AL amyloidosis (11\%). This suggests that early, tumor-initiating stages of malignant plasma cell development that lead to MGUS are more strongly controlled by the genetic susceptibility network than later stages of tumor promotion that yield frank neoplasia (MM or amyloidosis).

\section{MYELOMA RISK ALLELES DE- TECTED BY DNA SEQUENCING AND PCR-BASED GENOTYPING}

NGS and PCR-based genotyping provide additional approaches to myeloma risk allele discovery(Table 2). One example of the latter is the association of NCOAl with genetic susceptibility to myeloma in the Han Chinese population $^{[16]}$. NCOAl, one of three members of the p160/ SRC family 33 of proteins, acts as transcriptional coactivator for steroid and nuclear hormone receptors, but how this translates to myeloma risk is unclear. Other variants, which were discovered by PCR analysis to be involved in myeloma risk and therapy response, were found in $C R B N$ (cereblon) and IRF4 (interferon regulatory factor 4$)^{[17]}$. Variants of this sort can be added to a growing list of germline alleles that influence survival in myeloma; e.g., $B S G$ (basigin) and $M C T 1$ (monocarboxylate transporter 1, officially designated $S L C 16 A 1)^{[18]}, C D K N 2 A$ (cyclin dependent kinase inhibitor 2A) ${ }^{[19]}$, FOPNL (FGFR1OP $\mathrm{N}$-terminal like) ${ }^{[10]}$ and $A I C D A$ (activation induced cytidine deaminase $)^{[20]}$. NGS, particularly WES (whole exome sequencing) and exome sequencing in SGS (shared genomic segment) regions, affords a powerful method for uncovering myeloma risk alleles. Examples include ARIDIA (AT-rich interaction domain 1A) and USP45 (ubiquitin specific peptidase 45 ), which were detected in a pedigree analysis implicating DNA repair and chromatin remodeling in multiple myeloma risk $^{[21]}$, and $E P 300^{[22]}$, which encodes a histone acetyltransferase (HAT) known as p300 that regulates transcription via chromatin remodeling. Continuing with epigenetic regulators in the area of early-onset myeloma, WES recently identified germline $\mathrm{N}$-terminal truncating mutations in the first autosomal-dominant MM predisposition gene: $L S D I$ (lysine demethylase $1 \mathrm{~A}$, official gene symbol $K D M I A)^{[23]}$, which encodes a transcriptional repressor that primarily demethylates histone $\mathrm{H} 3$ on lysine 4. The finding that pharmacological inhibition of LSDI in antigenchallenged mice led to plasma cell expansion and the appearance of serum paraproteins supported the contention that the demethylase is involved in malignant plasma cell transformation ${ }^{[23]}$. WES followed by gene burden analysis additionally identi-

Table 2 Candidate myeloma susceptibility genes identified in NGS studies

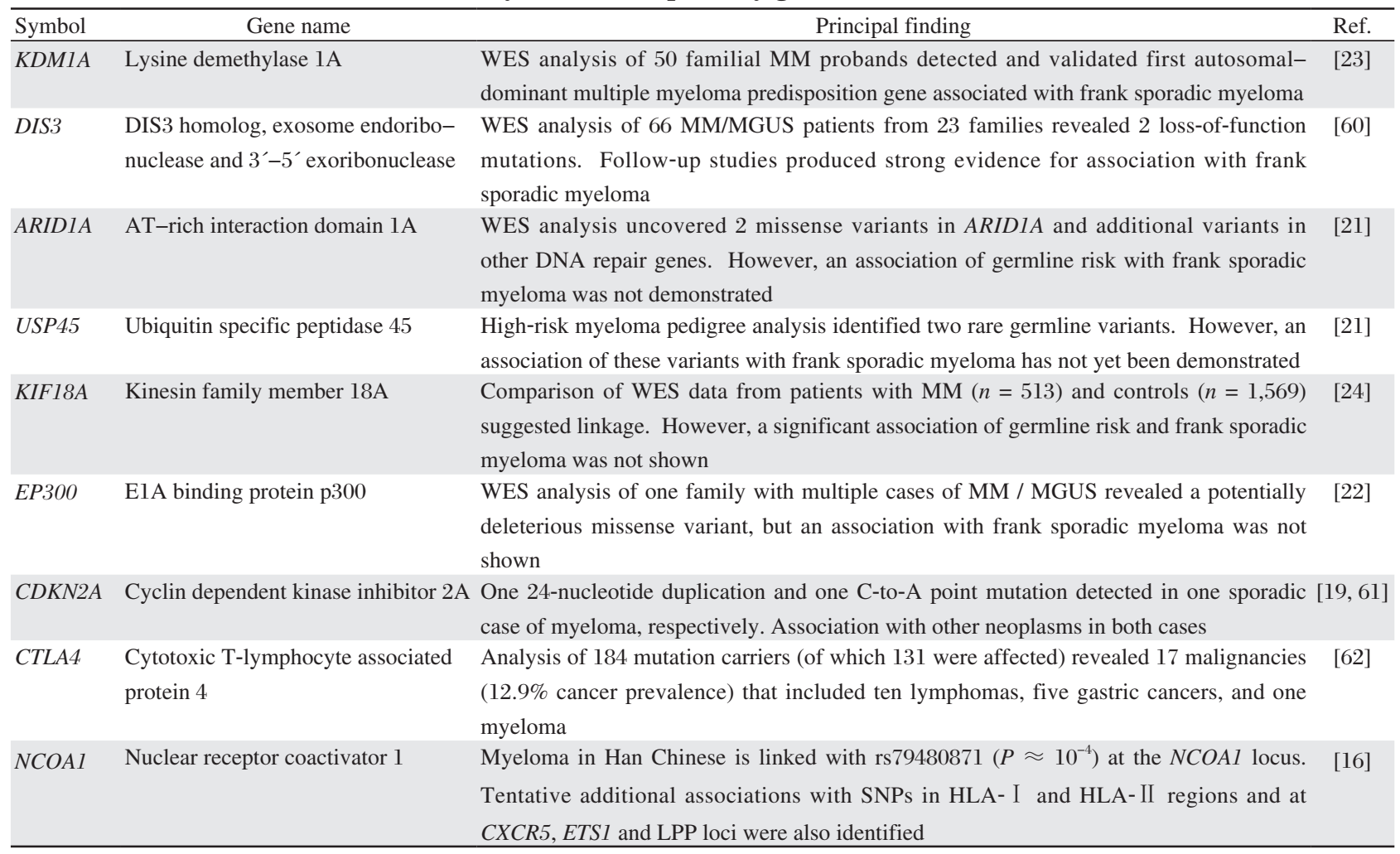


fied a candidate risk gene, $\operatorname{KIF18A}\left(P=3.6 \times 10^{-6}\right)$, that encodes a member of the kinesin superfamily of microtubule-associated molecular motors. KIF18A displays a distinct pattern of expression across molecular subgroups of MM and is associated with patient survival ${ }^{[24]}$. Last but not least, WES analysis of 66 cases of MM or MGUS in 23 unrelated families uncovered 2 loss-of-function mutations in DIS3, which encodes the catalytic subunit of the exosome complex, and frequently undergoes $(>10 \%)$ somatic mutagenesis in myeloma.

\section{MYELOMA RISK ALLELES UNCOV- ERED IN IMMUNOLOGICAL STUDIES}

The risk alleles identified by GWAS (Table 1) are common but of low impact, whereas the risk genes/alleles included in Table 2 are rare yet of relatively high impact. Nonetheless, the strongest risk factor for myeloma to date has not been identified in genetic but in immunological studies: the hyper-phosphorylated paratarg-7 ( $\mathrm{pP}-7)$ carrier state. The RR for $\mathrm{pP}-7$ carriers to develop MGUS/ MM is $7.9^{[25]}$. Paratarg, which is short for paraprotein target, refers to the circumstance that paraproteins frequently bind to protein and other antigens. Patients with a paraprotein that binds to paratarg-7 ( $\mathrm{P}-7$, which was later identified as stomatin-like protein 2 or STOML2) carry a hyper-phosphorylated form of $\mathrm{P}-7(\mathrm{pP}-7)$ that is inherited in an autosomal dominant manner. $\mathrm{pP}-7$ is found in over one third of MGUS/MM patients. Additional autoantigenic paraprotein targets were subsequently identified, all of which are hyper-phosphorylated in affected patients ${ }^{[26]}$. Hyper-phosphorylation may be the result of de-phosphorylation deficiency, based on the finding that de-phosphorylation of $\mathrm{pP}-7$ is defective in $\mathrm{pP}-7$ carriers due to low activity of protein-phosphatase $2 \mathrm{~A}^{[27]}$. The work on paratargs described above and exciting new research by Dhodapkar's group ${ }^{[28]}$ are consistent with the hypothesis that immune responses to post-translationally modified proteins and lipids may play a role in myeloma development. The association of human leukocyte antigen (HLA) polymorphism with myeloma risk lends further support to this vie ${ }^{[29]}$ because HLA proteins are instrumental in initiating $\mathrm{T}$ cell-dependent immune responses by virtue of presenting immunogenic peptides to the $\mathrm{T}$ cell receptor (TCR). Predisposing or protective associations of HLA polymorphisms with myeloma were identified at the level of individual HLA alleles (A, B, C, DRB3/4/5, DRB 1 and DQB1) and the level of haplotype combinations of these loci ${ }^{[29]}$.
The predisposing HLA region, for example, that was identified by Chubb et al. at 6p21.3 (Table 1) ${ }^{[6]}$ may represent $H L A-D R B 5^{*} 01^{[9]}$.

\section{RACIAL AND ETHNIC DIFFERENCES IN MYELOMA}

In the United States, the prevalence of MGUS and frank myeloma is significantly higher in African Americans (AA) than in Caucasian Americans (CA) of European ancestry ${ }^{[30]}$. For example, a study reporting that the overall myeloma incidence (cases per 100,000 persons) increased from 5.52 in the 5 -year period from 1993 to 1997 to 6.08 in the 2008-2012 period $(P<0.001)$ found an increase of $\sim 13 \%$ in CA men $(6.39-7.22 ; P<0.001)$ but an increase of $\sim 17 \%$ in AA men (13.94-16.15; $P$ $<0.01)$. Thus, in 2012 the myeloma incidence in AA men $\left(16.2 \times 10^{-5}\right)$ was 2.24 times higher than in CA men $\left(7.22 \times 10^{-5}\right)$ and the trend of disparity was increasing ${ }^{[31]}$. Another well-established racial difference is the mean age of diagnosing myeloma: it is 4 years younger in AA patients (65.8 years) compared to CA patients (69.8 years) ${ }^{[32]}$. Although confounding effects due to inequalities in health care and a host of environmental and lifestyle factors cannot be excluded, both the higher rate and earlier onset of MM in African Americans support the notion of a racial contribution to the etiology and natural history of MM. Realizing that myeloma disparity research may be hampered by uncertainty and bias introduced by self-reported race as opposed to objective genetic ancestry data, Rajkumar, Kumar and their associates took advantage of a genotyping tool dubbed Precision Medicine Research Array to determine biogeographical ancestry of myeloma patients in an unbiased, quantitative manner. Using this method, they were able to demonstrate that a major proportion of the racial AA vs. CA disparity in myeloma is driven by differences in the occurrence of myeloma-associated $\mathrm{t}(11 ; 14), \mathrm{t}(14 ; 16)$ and $\mathrm{t}(14 ; 20)$ translocations ${ }^{[33]}$. However, genetic myeloma risk is also involved, as discussed in greater depth in the following.

\section{RACIAL AND ETHNIC DIFFERENC- ES IN MYELOMA RISK}

To determine myeloma susceptibility regions for AA and CA individuals in greater depth, Cozen et al. performed a GWAS meta-analysis that included a clever imputation-based fine mapping approach to identify functional variants that govern myeloma risk $^{[34]}$. The study relied on several loci associated with myeloma risk (Table 1), including variants in 
ULK4 (unc-51 like kinase 4); a missense variant in TNFRSF13B, which encodes a B cell activating factor (BAFF) receptor from the TNF receptor family called TACI (transmembrane activator and calcium-modulating cyclophilin ligand interactor); SNPs around the promoter and enhancer regions of CBX7 (chromobox 7); and, importantly, a SNP at $7 p 15.3$ (rs4487645) that was independently confirmed in a GWAS that also implicated the $2 q 12.3$ region in myeloma risk ${ }^{[35]}$. The $7 p 15.3$ rs4487645 locus exhibits stronger association with $\mathrm{MM}$ in AA individuals compared to CA individuals ${ }^{[34]}: 0.89$ vs. $0.70 \mathrm{RAF}$ and $1.37 \mathrm{vs.} 1.23 \mathrm{OR}$ (at $99 \%$ power in both cases and $P$ values of $8.30 \times 10^{-5}$ for AA samples and $7.47 \times 10^{-4}$ for CA samples). To gain insight into the biological function of the $7 p 15.3$ (rs4487645) risk locus in myeloma, Weinhold, Hemminki and colleagues carried out an expression quantitative trait locus (eQTL) analysis ${ }^{[36]}$ which showed that the $\mathrm{C}$ risk allele results in elevated CDCA7L (cell division cycle associated 7 like) expression compared to the A "non-risk" allele. Following up on that, Li et al. demonstrated that the $C$ risk allele-dependent increase in $C D C A 7 L$ expression must be attributed to the generation of an IRF4 binding site in the $7 \mathrm{p} 15.3$ enhancer ${ }^{[37]}$. This connected the germline risk of myeloma to a genetic pathway of great significance for myeloma biology: IRF4-MYC. Li et al. also showed that $C D C A 7 L$ mRNA levels may prognosticate survival of patients with myeloma. For example, in the GSE9782 trial, myeloma patients ( $n=265$ total) in the top quartile of $C D C A 7 \mathrm{~L}$ expression (measured in bone marrow plasma cells) exhibited significantly shorter overall survival than patients in the bottom quartile $(P=$ $3.1 \times 10^{-4}$; hazard ratio $[\mathrm{HR}]=2.3$ ). Wendy Cozen and her associates recently updated the metaanalysis mentioned above and demonstrated that African Americans in the top $10 \%$ of a newly constructed polygenic risk score exhibit an increase in the myeloma risk by $80 \%{ }^{[38]}$.

\section{RACIAL AND ETHNIC DIFFERENCES IN SOMATIC MUTATION PATTERNS}

A recent NGS study on tumor-acquired somatic mutations in myeloma reported new insights into racial differences between AA and CA patients ${ }^{[39]}$. This included the discovery of significant differences in mutation frequency in 17 genes, with as many as 15 of them (88\%) demonstrating a higher mutation frequency in AA than CA myeloma (Table 3). IRF4 may be of special interest for two reasons: it is recurrently mutated in CA $(3.2 \%)$ but not AA patients and it is linked to germline risk in the CDCA7L locus as described above. IRF4 is an important transcription factor in the hematopoietic system ${ }^{[40]}$ that was identified as a myeloma driver in tumors that carry the IRF4-activating chromosomal $\mathrm{t}(6 ; 14)(\mathrm{p} 25 ; \mathrm{q} 32)$ translocation ${ }^{[41]}$. IRF4 expression is inversely correlated with myeloma clinical outcome ${ }^{[42]}$ and IRF4-dependent modulation of Fas-induced apoptosis governs in part myeloma survival ${ }^{[43]}$. What is more, studies on IRF4 target genes uncovered a positive auto-regulatory loop between IRF4 and $M Y C^{[44]}$. IRF $4^{\mathrm{K} 123 \mathrm{R}}$ is the most

Table 3 Genes exhibiting different somatic mutation rates in African American (AA) and Cau casian American (CA) patients with multiple myeloma

\begin{tabular}{|c|c|c|c|c|c|}
\hline Gene symbo $^{1}$ & Gene name & $\begin{array}{c}\text { Mutated in } \\
\text { AA }(\%)\end{array}$ & $\begin{array}{c}\text { Mutated in } \\
\text { CA }(\%)\end{array}$ & $\begin{array}{c}\text { AA vs. CA } \\
\text { ratio }\end{array}$ & $P$ value \\
\hline$A B I 3 B P$ & ABI family member 3 binding protein & 3.9 & 1.0 & 3.9 & 0.015 \\
\hline AUTS2 & Activator of transcription and developmental regulator & 3.9 & 1.2 & 3.3 & 0.028 \\
\hline$B C L 7 A$ & BCL tumor suppressor 7A & 3.9 & 0.8 & 4.9 & 0.007 \\
\hline BRWD3 & Bromodomain and WD repeat domain containing 3 & 3.9 & 0.8 & 4.9 & 0.007 \\
\hline$D D X 17$ & DAED-box helicase 17 & 3.1 & 0.7 & 4.4 & 0.016 \\
\hline$G R M 7$ & Glutamate metabotropic receptor 7 & 3.9 & 1.0 & 3.9 & 0.015 \\
\hline IRF4 & Interferon regulatory factor 4 & ND & 3.2 & N/A & 0.041 \\
\hline MYH13 & Myosin heavy chain 13 & 3.9 & 0.8 & 4.9 & 0.007 \\
\hline PARP4 & Poly (ADP-ribose) polymerase family member 4 & 3.9 & 1.0 & 3.9 & 0.015 \\
\hline PLDl & Phospholipase D1 & 3.1 & 0.3 & 10 & 0.002 \\
\hline PTCHD3 & Patched domain containing 3 & 4.7 & 1.0 & 4.7 & 0.003 \\
\hline$R Y R l$ & Ryanodine receptor 1 & 9.4 & 4.9 & 1.9 & 0.045 \\
\hline$S P E F 2$ & Sperm flagellar 2 & 3.9 & 0.8 & 4.9 & 0.001 \\
\hline$S T X B P 4$ & Syntaxin binding protein 4 & 3.1 & ND & N/A & $<10^{-3}$ \\
\hline TP53 & Tumor protein p53 & 1.6 & 6.3 & 0.25 & 0.035 \\
\hline
\end{tabular}

ND, not detected. N/A, not applicable. 
common mutant allele in myeloma ${ }^{[39,45]}$, with the resulting lysine-to-arginine exchange in the IRF domain of the protein constituting a putative gainof-function mutation. Somewhat paradoxically, however, Walker et al. recently reported that this mutation (and other exonic mutations in the gene body) results in improved survival in myeloma ${ }^{[45]}$. The reason for that is not clear but may be related to the possibility that tumor progression alleles such as $I R F 4^{\mathrm{K} 123 \mathrm{R}}$ sometimes improve the treatment response. IRF4 is also of great interest from the therapeutic angle since it constitutes a "unifying Achilles heel" in myeloma, regardless of molecular subtypes $^{[44]}$. For example, the backbone myeloma drug lenalidomide (Len) down regulates IRF4 indirectly by virtue of targeting cereblon (CRBN), the proximal regulator of the CRBN-IKFZ1/3IRF4-MYC pathway ${ }^{[46-50]}$.

\section{PROMISE}

A big step towards enhanced understanding of racial disparity in myeloma is the PROMISE study (NCT03689595), which is funded as part of the United States National Cancer Institute Stand Up To Cancer Multiple Myeloma Dream Team. The acronym PROMISE stands for Predicting Progression of Developing Myeloma in a High-Risk Screened Population. The study will enroll an estimated 50,000 participants between 45 and 75 years of age, that are either AA individuals (selfidentified) or individuals of any race who have a first-degree relative (parent, sibling or child) with frank myeloma or the precursor conditions MGUS and smoldering multiple myeloma (SMM). The $\mathrm{IgM}^{+}$plasma cell dyscrasia, Waldenström macroglobulinemia, will also be accepted as inclusion criterion. The completion of PROMISE, which is poised to close long-standing knowledge gaps on early stages of myeloma development, is envisioned for 2033. The primary outcome measure is time to progression (TTP) from MGUS/SMM to frank myeloma. The principal goal of the study is the definition of the clinical, (epi) genetic, genomic and/or immune environmental parameters that predict progression to overt cancer. PROMISE is coled by Drs. Irene M. Ghobrial and Ivan M. Borrello from the Dana Farber Cancer Institute, Boston, Massachusetts and Johns Hopkins School of Medicine, Baltimore, Maryland, respectively. The study will not only address the high burden of myeloma in the African American population but will also catalyze fresh thinking about how to make myelo- ma a preventable disease.

\section{KEY POINTS AND FUTURE DIRECTIONS}

GWAS has identified 24 common but lowimpact myeloma risk loci (Table 1) that, taken together, explain approximately one eighth of the heritable myeloma risk in Caucasians. The risk of myeloma overlaps with that of MGUS and AL amyloidosis. WES analysis of MM / MGUS kindreds has additionally identified a handful of rare but high-impact myeloma risk loci (Table 2) that provide intriguing clues about myeloma etiology. The Chinese myeloma community is now challenged with complementing these results in Western populations with data on Han Chinese and the many smaller ethnic groups that live in the People's Republic of China. A good starting point to that end may be the analysis of MM / MGUS kindreds in the PRC. A useful blueprint for the recruitment of kindreds for germline mutation studies on myeloma risk has been published just recently by an investigation team in Germany ${ }^{[51]}$. Equally important for progress is the continuation of the line investigation that led to the discovery of NCOAl as a myeloma susceptibility gene in Han Chinese ${ }^{[16]}$. The incidence of myeloma in the PRC is significantly lower than in Western countries, suggesting that major differences in GWAS-identifiable germline risk loci exist. Liu et al. recently reported an estimated 16,500 new myelomas in China in $2016^{[52]}$, which translates to an age-standardized incidence rate of $\sim 1$ per 100,000 people. However, this number should be taken with a grain of salt because the burden of MM in the PRC exhibits stark contrasts at the provincial level and additional research must be carried out before the incidence of myeloma in China can be fully evaluated.

Going forward, the international myeloma community in close partnership with its Chinese colleagues is tasked with validating myeloma risk loci at the functional level. This process has already started, using $E L L 2^{[53,54]}, C D C A 7 L^{[36,37]}$ and $C C N D 1^{[5]}$ as study objects, but much more needs to be done to complete the picture. In parallel to gene-centric studies, genetic network analyses should be performed to integrate myeloma risk alleles in functional pathways. Themes along this line that have recently emerged include B cell and plasma cell development (TNFRSF13B, ATG5, ELL2, CBX7, KLF2, and HLA region), autophagy (WAC, ULK4, TOM1), telomere maintenance (POT1, 
TERC) and cell cycle regulation and DNA replication (CDCA7L, CDKN2A, CCND1, RFWD3) ${ }^{[9]}$. Another area of future work on genetic myeloma risk concerns the flip side of susceptibility: genetic resistance. The first myeloma resistance gene, LIG4 (rs1555902), has been recently identified ${ }^{[20]}$ but many more remain to be discovered. Elucidation of myeloma resistance may inspire new approaches to myeloma prevention. A third research area that warrants more attention is gene-environment interaction, exemplified by the possible link of occupational exposure to cholinergic agents (pesticides) with myeloma incidence and myeloma risk $^{[55,56]}$. An earlier example is the association of genetic variations in the benzene metabolism with myeloma risk ${ }^{[57]}$. The biological effect of many susceptibility/resistance genes may depend, at least in part, on specific environmental or occupational exposures. An implicit promise of all these efforts is the discovery of novel molecular targets for myeloma treatment and prevention. New biomarkers for improved patient management in the clinic, including individualized myeloma treatment plans, may also be brought to light.

\section{Acknowledgements and funding}

This work was supported by the MCW Milwaukee William G. Schuett, Jr., Multiple Myeloma Research Endowment. Additional support was provided by NIH grant R01CA151354 to SJ.

\section{References}

[1] Kristinsson SY, Bjorkholm M, Goldin LR, et al. Patterns of hematologic malignancies and solid tumors among 37,838 first-degree relatives of 13,896 patients with multiple myeloma in Sweden[J]. Int $J$ Cancer, 2009, 125(9): 2147-50.

[2] Morgan GJ, Johnson DC, Weinhold N, et al. Inherited genetic susceptibility to multiple myeloma[J]. Leukemia, 2014, 28(3): 518-24.

[3] Broderick P, Chubb D, Johnson DC, et al. Common variation at 3 p22.1 and 7 p15.3 influences multiple myeloma risk[J]. Nat Genet, 2011, 44(1): 58-61.

[4] Martino A, Campa D, Jamroziak K, et al. Impact of polymorphic variation at $7 \mathrm{p} 15.3,3 \mathrm{p} 22.1$ and $2 \mathrm{p} 23.3$ loci on risk of multiple myeloma[J]. Br J Haematol, 2012, 158(6): 805-9.

[5] Weinhold N, Johnson DC, Chubb D, et al. The CCND1 c.870G $>$ A polymorphism is a risk factor for $\mathrm{t}(11 ; 14)(\mathrm{q} 13 ; \mathrm{q} 32)$ multiple myeloma[J]. Nat Genet, 2013, 45(5): 522-5.

[6] Chubb D, Weinhold N, Broderick P, et al. Common variation at $3 \mathrm{q} 26.2,6 \mathrm{p} 21.33,17 \mathrm{p} 11.2$ and $22 \mathrm{q} 13.1$ influences multiple myeloma risk[J]. Nat Genet,
2013, 45(10): 1221-5.

[7] Mitchell JS, Li N, Weinhold N, et al. Genome-wide association study identifies multiple susceptibility loci for multiple myeloma[J]. Nat Commun, 2016, 7: 12050.

[8] Went M, Sud A, Forsti A, et al. Identification of multiple risk loci and regulatory mechanisms influencing susceptibility to multiple myeloma[J]. Nat Commun, 2018, 9(1): 3707.

[9] Pertesi M, Went M, Hansson M, et al. Genetic predisposition for multiple myeloma[J]. Leukemia, 2020, 34(3): 697-708.

[10] Ziv E, Dean E, Hu D, et al. Genome-wide association study identifies variants at $16 \mathrm{p} 13$ associated with survival in multiple myeloma patients[J]. Nat Commun, 2015, 6: 7539 .

[11] Rajkumar SV, Merlini G, San Miguel JF. Haematological cancer: Redefining myeloma[J]. Nat Rev Clin Oncol, 2012, 9(9): 494-6.

[12] Weinhold N, Johnson DC, Rawstron AC, et al. Inherited genetic susceptibility to monoclonal gammopathy of unknown significance[J]. Blood, 2014, 123(16): 2513-7; quiz 2593.

[13] Thomsen H, Campo C, Weinhold N, et al. Genomewide association study on monoclonal gammopathy of unknown significance (MGUS)[J]. Eur J Haematol, 2017, 99(1): 70-9.

[14] Chattopadhyay S, Thomsen H, Weinhold N, et al. Eight novel loci implicate shared genetic etiology in multiple myeloma, AL amyloidosis, and monoclonal gammopathy of unknown significance[J]. Leukemia, 2020, 34(4): 1187-91.

[15] Chattopadhyay S, Thomsen H, da Silva Filho MI, et al. Enrichment of $\mathrm{B}$ cell receptor signaling and epidermal growth factor receptor pathways in monoclonal gammopathy of undetermined significance: a genome-wide genetic interaction study[J]. Mol Med, 2018, 24(1): 30 .

[16] Peng M, Zhao G, Yang F, et al. NCOAl is a novel susceptibility gene for multiple myeloma in the Chinese population: A case-control study[J]. PLoS One, 2017, 12(3): e0173298.

[17] Butrym A, Lacina P, Rybka J, et al. Cereblon and IRF4 variants affect risk and response to treatment in multiple myeloma[J]. Arch Immunol Ther Exp (Warsz), 2016, 64(Suppl 1): 151-6.

[18] Lacina P, Butrym A, Mazur G, et al. BSG and MCT1 genetic variants influence survival in multiple myeloma patients[J]. Genes (Basel), 2018, 9(5): 226.

[19] Shah V, Boyd KD, Houlston RS, et al. Constitutional mutation in CDKN2A is associated with long term survivorship in multiple myeloma: a case report[J]. BMC Cancer, 2017, 17(1): 718.

[20] Campa D, Martino A, Macauda A, et al. Genetic polymorphisms in genes of class switch recombination and multiple myeloma risk and survival: an IMMEnSE study[J]. Leuk Lymphoma, 2019, 60(7): 1803-11. 
[21] Waller RG, Darlington TM, Wei X, et al. Novel pedigree analysis implicates DNA repair and chromatin remodeling in multiple myeloma risk[J]. PLoS Genet, 2018, 14(2): e1007111.

[22] Bolli N, Barcella M, Salvi E, et al. Next-generation sequencing of a family with a high penetrance of monoclonal gammopathies for the identification of candidate risk alleles[J]. Cancer, 2017, 123(19): 3701-8.

[23] Wei X, Calvo-Vidal MN, Chen S, et al. Germline lysinespecific demethylase 1 (LSDl/KDMlA) mutations confer susceptibility to multiple myeloma[J]. Cancer Res, 2018, 78(10): 2747-59.

[24] Scales M, Chubb D, Dobbins SE, et al. Search for rare protein altering variants influencing susceptibility to multiple myeloma[J]. Oncotarget, 2017, 8(22): 36203-10.

[25] Grass S, Preuss KD, Ahlgrimm M, et al. Association of a dominantly inherited hyperphosphorylated paraprotein target with sporadic and familial multiple myeloma and monoclonal gammopathy of undetermined significance: a casecontrol study[J]. Lancet Oncol, 2009, 10(10): 950-6.

[26] Grass S, Preuss KD, Thome S, et al. Paraproteins of familial MGUS/multiple myeloma target family-typical antigens: hyperphosphorylation of autoantigens is a consistent finding in familial and sporadic MGUS/MM[J]. Blood, 2011, 118(3): 635-7.

[27] Preuss KD, Fadle N, Regitz E, et al. Inactivation of proteinphosphatase $2 \mathrm{~A}$ causing hyperphosphorylation of autoantigenic paraprotein targets in MGUS/MM is due to an exchange of its regulatory subunits[J]. Int J Cancer, 2014, 135(9): 2046-53.

[28] Nair S, Sng J, Boddupalli CS, et al. Antigen-mediated regulation in monoclonal gammopathies and myeloma[J]. JCI Insight, 2018, 3(8): 98259

[29] Beksac M, Gragert L, Fingerson S, et al. HLA polymorphism and risk of multiple myeloma[J]. Leukemia, 2016, 30(11): 2260-4.

[30] Greenberg AJ, Vachon CM, Rajkumar SV. Disparities in the prevalence, pathogenesis and progression of monoclonal gammopathy of undetermined significance and multiple myeloma between blacks and whites[J]. Leukemia, 2012, 26(4): 609-14.

[31] Costa LJ, Brill IK, Omel J, et al. Recent trends in multiple myeloma incidence and survival by age, race, and ethnicity in the United States[J]. Blood Adv, 2017, 1(4): 282-7.

[32] Waxman AJ, Mink PJ, Devesa SS, et al. Racial disparities in incidence and outcome in multiple myeloma: a populationbased study[J]. Blood, 2010, 116(25): 5501-6.

[33] Baughn LB, Pearce K, Larson D, et al. Differences in genomic abnormalities among African individuals with monoclonal gammopathies using calculated ancestry[J]. Blood Cancer J, 2018, 8(10): 96.

[34] Rand KA, Song C, Dean E, et al. A meta-analysis of multiple myeloma risk regions in African and European ancestry populations identifies putatively functional loci[J]. Cancer Epidemiol Biomarkers Prev, 2016, 25(12): 1609-18.

[35] Erickson SW, Raj VR, Stephens OW, et al. Genome-wide scan identifies variant in $2 \mathrm{q} 12.3$ associated with risk for multiple myeloma[J]. Blood, 2014, 124(12): 2001-3.

[36] Weinhold N, Meissner T, Johnson DC, et al. The 7p15.3 (rs4487645) association for multiple myeloma shows strong allele-specific regulation of the MYC-interacting gene CDCA7L in malignant plasma cells[J]. Haematologica, 2015, 100(3): el10-3.

[37] Li N, Johnson DC, Weinhold N, et al. Multiple myeloma risk variant at $7 \mathrm{pl} 5.3$ creates an IRF4-binding site and interferes with CDCA7L expression[J]. Nat Commun, 2016, 7: 13656.

[38] Du Z, Weinhold N, Song GC, et al. A meta-analysis of genome-wide association studies of multiple myeloma among men and women of African ancestry[J]. Blood Adv, 2020, 4(1): 181-90.

[39] Manojlovic Z, Christofferson A, Liang WS, et al. Comprehensive molecular profiling of 718 multiple myelomas reveals significant differences in mutation frequencies between African and European descent cases[J]. PLoS Genet, 2017, 13(11): el007087.

[40] Acquaviva J, Chen X, Ren R. IRF-4 functions as a tumor suppressor in early B-cell development[J]. Blood, 2008, 112(9): 3798-806.

[41] Iida S, Rao PH, Butler M, et al. Deregulation of MUM1/ IRF4 by chromosomal translocation in multiple myeloma[J]. Nat Genet, 1997, 17(2): 226-30.

[42] Heintel D, Zojer N, Schreder M, et al. Expression of MUM1/IRF4 mRNA as a prognostic marker in patients with multiple myeloma[J]. Leukemia, 2008, 22(2): 441-5.

[43] Fanzo JC, Yang W, Jang SY, et al. Loss of IRF-4-binding protein leads to the spontaneous development of systemic autoimmunity[J]. J Clin Invest, 2006, 116(3): 703-14.

[44] Shaffer AL, Emre NC, Lamy L, et al. IRF4 addiction in multiple myeloma[J]. Nature, 2008, 454(7201): 226-31.

[45] Walker BA, Boyle EM, Wardell CP, et al. Mutational spectrum, copy number changes, and outcome: Results of a sequencing study of patients with newly diagnosed myeloma[J]. J Clin Oncol, 2015, 33(33): 3911-20.

[46] Zhu YX, Braggio E, Shi CX, et al. Cereblon expression is required for the antimyeloma activity of lenalidomide and pomalidomide[J]. Blood, 2011, 118(18): 4771-9.

[47] Zhu YX, Braggio E, Shi CX, et al. Identification of cereblon-binding proteins and relationship with response and survival after IMiDs in multiple myeloma[J]. Blood, 2014, 124(4): 536-45.

[48] Greenberg AJ, Walters DK, Kumar SK, et al. Responsiveness of cytogenetically discrete human myeloma cell lines to lenalidomide: lack of correlation with cereblon and interferon regulatory factor 4 expression levels[J]. Eur J Haematol, 2013, 91(6): 504-13.

[49] Schuster SR, Kortuem KM, Zhu YX, et al. The clinical significance of cereblon expression in multiple myeloma[J]. Leuk Res, 2014, 38(1): 23-8.

[50] Lopez-Girona A, Heintel D, Zhang LH, et al. Lenalidomide downregulates the cell survival factor, interferon regulatory factor-4, providing a potential mechanistic link for predicting response[J]. Br J Haematol, 2011, 154(3): 325-36. 
[51] Blocka J, Durie BGM, Huhn S, et al. Familial cancer: how to successfully recruit families for germline mutations studies? Multiple myeloma as an example[J]. Clin Lymphoma Myeloma Leuk, 2019, 19(10): 635-44 e2.

[52] Liu J, Liu W, Mi L, et al. Incidence and mortality of multiple myeloma in China, 2006-2016: an analysis of the Global Burden of Disease Study 2016[J]. J Hematol Oncol, 2019, 12(1): 136.

[53] Ali M, Ajore R, Wihlborg AK, et al. The multiple myeloma risk allele at 5q15 lowers ELL2 expression and increases ribosomal gene expression[J]. Nat Commun, 2018, 9(1): 1649.

[54] Li N, Johnson DC, Weinhold N, et al. Genetic predisposition to multiple myeloma at $5 \mathrm{q} 15$ is mediated by an ELL2 enhancer polymorphism[J]. Cell Rep, 2017,
20(11): 2556-64.

[55] Shearer JJ, Beane Freeman LE, Liu D, et al. Longitudinal investigation of haematological alterations among permethrin-exposed pesticide applicators in the Biomarkers of Exposure and Effect in Agriculture study[J]. Occup Environ Med, 2019, 76(7): 467-70.

[56] Tual S, Busson A, Boulanger M, et al. Occupational exposure to pesticides and multiple myeloma in the AGRICAN cohort[J]. Cancer Causes Control, 2019, 30(11): 1243-50.

[57] Lincz LF, Scorgie FE, Robertson R, et al. Genetic variations in benzene metabolism and susceptibility to multiple myeloma[J]. Leukemia Res, 2007, 31(6): 759-63.

Received 29 February 2020, Revised 08 April 2020, Accepted 21 April 2020 